hep-th/9601050

BONN-TH-96-01

IFP-606-UNC

January 1996

\title{
Exactly Solvable Points in the Moduli Space of Heterotic $\mathrm{N}=2$ Strings
}

\author{
Ralph Blumenhagen ${ }^{1}$ and Andreas Wißkirchen ${ }^{2}$ \\ ${ }^{1}$ Institute of Field Physics, Department of Physics and Astronomy, \\ University of North Carolina, Chapel Hill NC 27599-3255, USA \\ 2 Physikalisches Institut der Universität Bonn, Nußallee 12, 53115 Bonn, Germany
}

\begin{abstract}
We investigate the subset of exactly solvable $(0,4)$ world sheet supersymmetric string vacua contained in a recent class of Gepner-like $(0,2)$ superconformal models. The identification of these models with certain points of enhanced gauge symmetry on $K_{3} \times T_{2}$ can be achieved completely. Furthermore, we extend the construction of in general $(0,2)$ supersymmetric exactly solvable models to the case where also a nontrivial part of the vector bundle is embedded into the hidden $E_{8}$ gauge group. For some examples we explicitly calculate the enhanced gauge symmetries and show that they open up the way to interesting branches of the $N=2$ moduli space. For some of these models candidates of type II dual descriptions exist.
\end{abstract}

1 e-mail: blumenha@physics.unc.edu

2 e-mail: wisskirc@avzw02.physik.uni-bonn.de 


\section{Introduction}

In two recent papers $[6,7]$ we have presented a class of exactly solvable heterotic string models with in general $(0,2)$ supersymmetry for the internal superconformal field theory (SCFT). The method used to construct these purely heterotic modular invariant partition functions was based on the simple current technique developed by Schellekens and Yankielowicz $[18,19,20,21]$ and independently by Intriligator [12]. Since the input data of a SCFT are completely different from the ones used in the (non)linear $\sigma$-model approach for strings in background fields, it turned out to be quite a difficult task to identify models from both sets. However, for at least three models such a one to one correspondence could be achieved by comparing the chiral rings of these $N=1$ space-time supersymmetric string vacua [7]. Therefore, we strongly believe that the class of $(0,2) \sigma$-models does really provide us with fully fledged classical vacua of the string disproving, at least for certain points in the moduli space, earlier indications that they are destabilized by world sheet instanton effects.

During the last months heterotic models with $N=2$ space-time supersymmetry have received wide attention due to their relevance for a stringy generalization of the Seiberg/Witten duality for $N=2$ Yang-Mills theories [22]. There exist convincing arguments that some heterotic strings on $K_{3} \times T_{2}$ admit a dual description in terms of a type II A string compactified on a Calabi-Yau threefold $[2,3,9,13,14,15,24]$ with the special property of being a $K_{3}$ fibration $[4,16]$. Nonperturbative topology changing transitions between different Calabi-Yau moduli spaces $[5,11,23]$ for the type II string correspond on the heterotic side to the existence of points of enhanced gauge symmetry at which new Higgs phases appear. We will address several questions in this letter. First, we will construct exactly solvable SCFTs with $N=2$ space-time supersymmetry. Since there is only one suitable manifold for the compactification, an identification of our models can be carried out much easier compared to the $N=1$ case, for one only has to find the vector bundle on $K_{3}$ for the left moving $\sigma$-model fermions. Furthermore, from earlier calculations it is known that exactly solvable SCFTs usually correspond to points in the moduli space where enhanced gauge symmetries occur. For instance, the usual Gepner models [10] have at least a further $U(1)^{r-1}$ piece in the gauge group. We will construct and analyze SCFTs, for which also nonabelian groups occur. By the Higgs mechanism we can break parts of the enhanced gauge symmetry and arrive at models for which a dual type II string appears in the lists of $K_{3}$ fibrations in [16,17]. A similar calculation has been carried out in [1] using orbifold techniques for $T_{4} / Z_{M} \times T_{2}$. Thus, our results can be considered as the generalization of their analysis to Gepner-like SCFTs.

It is clear that as long as the hidden gauge group $E_{8}$ is not broken one can only achieve a very limited set of models, for the number of generic vector multiplets is forced to be larger than nine. Thus, we will further address the problem, how the embedding of a vector bundle into the hidden $E_{8}$ factor can be implemented into the modular invariant partition functions constructed in [6]. An identification of these models with certain bundles on $K_{3}$ is straightforward and the Higgs phase of such models provides us with a lot of type II duals. 


\section{Exactly solvable $\mathrm{N}=2$ string models}

It is well known that in order to get $N=2$ space-time supersymmetry in the heterotic string compactified to four dimensions one needs a toroidal $c=3$ piece and at least a $(0,4)$ world sheet supersymmetric $c_{r}=6$ piece which corresponds to the $K_{3}$ manifold. The left moving central charge $c_{l}$, however, can vary reflecting the freedom of choosing vector bundles of arbitrary rank for the left moving $\sigma$-model fermions.

In this section we will first calculate a bunch of examples of exactly solvable $(0,4)$ models with generic $E_{7} \times E_{8}$ gauge group and by comparing the massless spectra we will try to identify them with suitable bundles on $K_{3}$. To this end, we review how the massless spectrum is determined for general bundles on $K_{3}$.

The unbroken gauge group in ten dimensions should be $G=E_{8} \times E_{8}$. Now, one embeds a (complexified) stable, holomorphic vector bundle $V$ into $\mathcal{G}$, which breaks it down to a subgroup $G$ such that $G \times H \subset \mathcal{G}$ is the maximal subalgebra. Then one can calculate the massless matter spectrum as follows: decompose

$$
\operatorname{adj}(\mathcal{G})=\sum_{i}\left(M_{i}, R_{i}\right)
$$

where $M_{i}$ and $R_{i}$ are representations of $G$ and $H$, respectively. Then, the number of left-handed spinor multiplets transforming in the $M_{i}$ representation of $G$ is given by the Atiyah-Singer index theorem:

$$
N_{M_{i}}=\operatorname{dim}\left(R_{i}\right)-\frac{1}{2} \int_{K_{3}} c_{2}(V) \operatorname{index}\left(R_{i}\right) .
$$

Furthermore, there appear 20 gauge singlet hypermultiplet moduli arising from higher dimensional gravitational fields. For the $S U(2)$ tangent bundle on $K_{3}$ one has $\int_{K_{3}} c_{2}(V)=$ 24. If one first compactifies the ten-dimensional theory on a torus, there exists the possibility of embedding vector bundles $V_{a}$ also into the possibly extended gauge group factors of the torus, $S U(3)_{T}, S U(2)_{T}^{2}$ and $S U(2)_{T}$. Then the following conditions have to be satisfied:

$$
c_{1}\left(V_{a}\right)=0, \quad \sum_{a} c_{2}\left(V_{a}\right)=c_{2}\left(T K_{3}\right)=24
$$

so that the bundles admit spinors and satisfy the Donaldson-Uhlenbeck-Yau equation and the anomaly cancellation condition, respectively. Using our stochastic search computer program for the construction of exactly solvable models with $N=2$ space-time supersymmetry we find a lot of models with all kinds of gauge groups. For instance, we obtain a model with gauge group $S O(10) \times E_{8}$ where the vacuum sector contains $N_{g}=15$ further massless vectors ${ }^{1}$, which also form a to be determined gauge group $G(13) \times U(1)_{T}^{2}$ of dimension 15. Furthermore, the massless sector contains $N_{16}=10$ hypermultiplets

1 Actually, there are 14 vector multiplets including the dilaton multiplet plus the graviphoton from the graviton multiplet. The graviphoton and the vector in the dilaton multiplet form a $U(1)_{T}^{2}$ part of the gauge group. 
in the spinor representation, $N_{10}=12$ hypermultiplets in the vector representation and $N_{1}=100$ hypermultiplets in the singlet representation of $S O(10)$. On the one hand side, by embedding an $S U(4)$ bundle with $A_{4}^{E_{8}}=\int_{K_{3}} c_{2}(V)=18$ into $E_{8}$ and an $S U(3)$ bundle with $A_{3}^{S U(3)}=6$ into the enhanced toroidal $S U(3)_{T}$ gauge group one gets the gauge group $G^{\prime}=S O(10) \times E_{8} \times U(1)_{T}^{2}$ with the following massless spectrum: $N_{16}=10, N_{10}=12$, $N_{1}=87$. By giving vacuum expectation values (VEVs) to exactly $r=13$ of our hypermultiplet moduli we can break the enhanced gauge group of dimension 15 down to $U(1)_{T}^{2}$ and thus reaching complete agreement of the two spectra. On the other hand side, this model can be understood as lying in the following pattern of successive symmetry breakings:

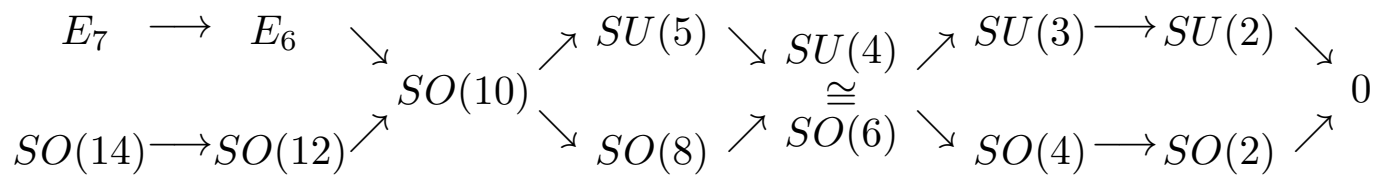

The corresponding $E_{7}$ model has $N_{56}=7$ and $N_{1}=76$ hypermultiplets and also a further gauge group of dimension $N_{g}=15$.

We find another example with gauge group $E_{8} \times E_{8} \times U(1)_{T}^{2} \times G(10)$ and $N_{1}=84$ hypermultiplets in the singlet representation. This model can be understood by embedding an $S U(3)$ bundle with $A_{3}^{S U(3)}=24$ into the toroidal $S U(3)_{T}$.

In Table 2.1 we enumerate the interpretation in terms of bundles on $K_{3}$ for some of the exactly solvable models we obtained. We always list only the $E_{7}$ parent model and indicate in column six at which special level in the cascade of symmetry breaking our exactly solvable model occurs.

\begin{tabular}{|l|l|l|l|l|l|l|}
\hline$N_{56}$ & $N_{1}$ & $N_{g}$ & bundle & enhanced gauge group & $G^{\prime}$ & $r$ \\
\hline 10 & $65+r$ & $10+r$ & $A_{2}^{E_{8}}=24$ & $G^{\prime} \times S U(3)_{T} \times U(1)_{T}^{2} \times G(r)$ & $E_{7}, E_{6}, S O(12)$ & $9,6,4,2$ \\
\hline 8 & $62+r$ & $3+r$ & $\begin{array}{l}A_{2}^{E_{8}}=20 \\
A_{2}^{S U(2)}=4\end{array}$ & $G^{\prime} \times U(1)_{T}^{3} \times G(r)$ & $S O(12), S O(10)$ & $\begin{array}{l}19,14,10 \\
7,5\end{array}$ \\
\hline 7 & $63+r$ & $2+r$ & $\begin{array}{l}A_{2}^{E_{8}}=18 \\
A_{3}^{S U(3)}=6\end{array}$ & $G^{\prime} \times U(1)_{T}^{2} \times G(r)$ & $E_{6}, S O(10)$ & $13,8,5$ \\
\hline 6 & $65+r$ & $2+r$ & $\begin{array}{l}A_{2}^{E_{8}}=16 \\
A_{3}^{S U(3)}=8\end{array}$ & $G^{\prime} \times U(1)_{T}^{2} \times G(r)$ & $S O(12)$ & 14 \\
\hline 4 & $69+r$ & $2+r$ & $\begin{array}{l}A_{2}^{E_{8}}=12 \\
A_{3}^{S U(3)}=12\end{array}$ & $G^{\prime} \times U(1)_{T}^{2} \times G(r)$ & $E_{7}, E_{6}$ & $17,13,6$ \\
\hline 2 & $59+r$ & $2+r$ & $\begin{array}{l}A_{2}^{E_{8}}=8 \\
A_{2,2}^{S U(2)}=16\end{array}$ & $G^{\prime} \times U(1)_{T}^{2} \times G(r)$ & $S O(14)$ & 21 \\
\hline 1 & $75+r$ & $2+r$ & $\begin{array}{l}A_{2}^{E_{8}}=6 \\
A_{3}^{S U(3)}=18\end{array}$ & $G^{\prime} \times U(1)_{T}^{2} \times G(r)$ & $E_{7}$ & 7 \\
\hline
\end{tabular}

Table $2.1 \quad$ Exactly solvable $N=2$ spectra 
Note that the appearance of the exceptional $S U(2)_{T} \times U(1)_{T}$ gauge group for the torus in the $N_{56}=8$ example is not obvious at all from the numbers in Table 2.1, for they would also fit with an $S U(3)_{T}$ gauge group. However, as we will see in the following section, the massless spectrum does indeed contain a further $U(1)$ current, relative to which all other fields are uncharged. Certainly, our list of $(0,4)$ models obtainable from the $(0,2)$ generalized Gepner models is not complete. However, it already shows that probably all models would fit into the scheme of $K_{3}$ compactifications. Thus we are confident that this nice picture will also hold for all $N=1$ supersymmetric SCFTs, even though a case by case identification with bundles on arbitrary Calabi-Yau threefolds is hardly to reveal.

\section{Special points of enhanced gauge symmetry}

After identifying exactly solvable $(0,4)$ string models with certain bundles on $K_{3} \times T_{2}$ we draw our attention to the complete gauge symmetry of these models. In the Gepner case this gauge group is in most cases only a further $U(1)^{r-1}$ abelian group. However, we will see that this is not necessarily the case if one has introduced further simple currents in the modular invariant partition function. The motivation is, that points of enhanced symmetry turned out to be an important issue in the study of how the moduli space of heterotic $N=2$ strings is connected. By giving VEVs to various kinds of scalar fields both in the hypermultiplets and the vector multiplets one can move from one branch of the moduli space to another one thereby changing the spectrum $\left(n_{H}, n_{V}\right)$ drastically. Thus, in order to gain information about the points of enhanced symmetry on $K_{3}$, one can use SCFTs as a natural source for such points.

In [13] it was argued that some heterotic $N=2$ models have a dual description as type II A models compactified on a Calabi-Yau manifold. Since in the type II A string the dilaton is a member of a hypermultiplet, the spectrum is given by $\left(n_{H}, n_{V}\right)=\left(b_{21}+\right.$ $\left.1, b_{11}+1\right)$. If such a duality holds one can calculate the full quantum moduli space of the vector multiplets on the type II A side and the quantum moduli space of the hypermultiplets on the heterotic side. Thus, analogously to mirror symmetry one has a handle on the complete quantum corrected field theory by using this complementary picture.

\section{Example based on the $(1)^{3}(4)^{3}$ parent}

In the following we will study in detail some of the models in the lists above. We start with the model with gauge symmetry $S O(10) \times E_{8} \times U(1)_{T}^{2} \times G(13)$ and the massless spectrum $N_{16}=10, N_{10}=12$ and $N_{1}=100$. Inspection of the couplings of the right moving vacuum in the modular invariant partition function shows that besides the $6 U(1)$ currents from each of the unitary factors there are exactly seven further currents around. All these together can be shown to form an $S U(3)_{E} \times U(1)_{E}^{5}$ algebra, so that the fully enhanced gauge symmetry at this point of the moduli space is

$$
S O(10) \times E_{8} \times S U(3)_{E} \times U(1)_{E}^{5} \times U(1)_{T}^{2} .
$$

After we have determined the complete enhanced gauge symmetry of the model we have to show how the massless sector fits into irreducible representations of $S O(10) \times S U(3)_{E}$. The answer is as follows:

$$
(16,3)+7 \times(16,1)+(10,3)+9 \times(10,1)+28 \times(1,3)+16 \times(1,1) .
$$


We have suppressed all the $U(1)_{E}^{5}$ charges, where for every $U(1)_{E}$ factor there exists at least one state carrying nonzero charge under this current. Thus, they can be higgsed away completely.

The information in (3.2) allows us to investigate the Higgs sector of the model by successively giving VEVs to some fields in the fundamental representation of the gauge group such that it is broken down to some subgroup. Then, by giving VEVs to the adjoint representation of the remaining gauge group we move to a generic point in the vector moduli and the massless spectrum is given by two numbers, the number of hypermultiplets and the number of vector multiplets. In Table 3.1 we collect all models which occur in the Higgs phase of this model and have an appropriate type II A dual in the lists of $[16,17]$. These are approximately $6 \%$ of all spectra occurring in the entire chain. The second column features the remaining enhanced gauge group of the model before moving to a generic point in the vector moduli.

\begin{tabular}{|l|l|l|}
\hline$\left(n_{H}, n_{V}\right)$ & $G^{\prime} \subset S O(10) \times E_{8} \times S U(3)_{E} \times U(1)_{E}^{5} \times U(1)_{T}^{2}$ & $K_{3}$ fibration \\
\hline \hline$(322,10)$ & $E_{8} \times U(1)_{T}^{2}$ & $\mathbb{P}_{1,1,6,16,24}[48]$ \\
$(221,11)$ & $S U(2) \times E_{8} \times U(1)_{T}^{2}$ & $\mathbb{P}_{1,1,6,10,18}[36]$ \\
$(168,12)$ & $S U(3) \times E_{8} \times U(1)_{T}^{2}$ & $\mathbb{P}_{1,1,6,10,12}[30]$ \\
$(129,13)$ & $S U(4) \times E_{8} \times U(1)_{T}^{2}$ & $\mathbb{P}_{1,1,6,10,12,14}[2420]$ \\
\hline$(144,12)$ & $S U(2) \times E_{8} \times S U(2)_{E} \times U(1)_{T}^{2}$ & $\mathbb{P}_{1,1,6,8,10}[26]$ \\
\hline$(55,13)$ & $S O(2) \times E_{8} \times S U(3)_{E} \times U(1)_{T}^{2}$ & $\mathbb{P}_{2,2,5,5,6}[20]$ \\
$(50,14)$ & $S O(4) \times E_{8} \times S U(3)_{E} \times U(1)_{T}^{2}$ & $\mathbb{P}_{5,5,8,12,30}[60]$ \\
$(33,21)$ & $S O(8) \times E_{8} \times S U(3)_{E} \times U(1)_{E}^{5} \times U(1)_{T}^{2}$ & $\mathbb{P}_{3,3,4,4,10}[24]$ \\
\hline
\end{tabular}

Table 3.1 Heterotic/type II A duals

The cascade of the first four models has already been found in [1]. However, the second four models make use of the special enhanced gauge group $S U(3)_{E} \times U(1)_{E}^{5}$ and therefore lead to new branches of the $N=2$ moduli space.

\section{Example based on the (1) $(4)^{4}$ parent}

As a second example we study the model with gauge group $S O(10) \times E_{8} \times U(1)_{T}^{2} \times G(8)$ and the massless spectrum $N_{16}=12, N_{10}=14$ and $N_{1}=97$. By looking at the couplings to the right moving vacuum state, one realizes that there appears an enhanced $S U(2)_{E} \times U(1)$ gauge group, where the entire massless spectrum contains no state charged under this $U(1)$. This indicates that this model is actually the one where the torus gauge group is $S U(2)_{T} \times U(1)_{T}^{3}$ with a bundle with $A_{2}^{S U(2)}=4$ embedded into the $S U(2)_{T}$ part. The massless spectrum fits into the following representations of $S O(10) \times S U(2)_{E}$ :

$$
\begin{aligned}
& 4 \times(16,2)+4 \times(16,1)+(10,3)+4 \times(10,2)+3 \times(10,1)+ \\
& 4 \times(1,4)+11 \times(1,3)+16 \times(1,2)+16 \times(1,1)
\end{aligned}
$$

which is somehow surprising for the appearance of higher spin $S U(2)$ representations. Thus, appropriate simple currents give rise to the existence of higher level Kac-Moody algebras in the spectrum, in this case $S U(2)_{3}$. 
As in the previous example, we want to see what happens in the different Higgs phases of this model which are summarized in Table 3.2.

\begin{tabular}{|l|l|l|}
\hline$\left(n_{H}, n_{V}\right)$ & $G^{\prime} \subset S O(10) \times E_{8} \times S U(2)_{E} \times U(1)_{E}^{4} \times U(1)_{T}^{3}$ & $K_{3}$ fibration \\
\hline$(377,11)$ & $E_{8} \times U(1)_{T}^{3}$ & $\mathbb{P}_{1,1,8,20,30}[60]$ \\
$(252,12)$ & $S U(2) \times E_{8} \times U(1)_{T}^{3}$ & $\mathbb{P}_{1,1,8,12,22}[44]$ \\
$(187,13)$ & $S U(3) \times E_{8} \times U(1)_{T}^{3}$ & $\mathbb{P}_{1,1,8,12,14}[36]$ \\
$(140,14)$ & $S U(4) \times E_{8} \times U(1)_{T}^{3}$ & $\mathbb{P}_{1,1,8,12,14,16}[2824]$ \\
$(117,15)$ & $S O(8) \times E_{8} \times U(1)_{T}^{3}$ & $\mathbb{P}_{3,3,8,28,42}[84]$ \\
\hline
\end{tabular}

Table 3.2 Heterotic/type II A duals

The first four models were also found in [1].

\section{Example based on the $(1)^{3}(4)^{3}$ parent}

We briefly discuss a third example with gauge group $S O(12) \times E_{8} \times S U(3)_{E} \times S U(2)_{E} \times$ $U(1)_{E}^{3} \times U(1)_{T}^{2}$ at the exactly solvable point. The massless sector fits into the following representations of $S O(12) \times S U(3)_{E} \times S U(2)_{E}$ :

$$
\begin{aligned}
& (32,1,2)+2 \times(32,1,1)+2 \times(12,3,1)+2 \times(12,1,2)+2 \times(12,1,1)+ \\
& 4 \times(1,3,2)+10 \times(1,3,1)+4 \times(1,1,2)+14 \times(1,1,1) .
\end{aligned}
$$

The only possible candidate for a type II A dual model is reached by first higgsing the $S U(2)_{E} \times U(1)_{E}^{3}$ away, then move to generic point in the $S U(3)_{E}$ vector moduli and break the $S O(12)$ down to $S U(2)$. This yields at generic points in the $S U(2)$ moduli the spectrum $\left(n_{H}, n_{V}\right)=(97,13)$ which could be dual to the type II A string compactified on the $K_{3}$ fibration $\mathbb{P}_{1,1,6,8,8,10}[1816]$.

Summarizing, by studying different branches of the $N=2$ moduli space of these 3 special models we already found several possible candidates for heterotic duals of type II A strings. One apparent limitation is that so far we have not broken the hidden $E_{8}$ symmetry in the exactly solvable approach, thus $n_{V}$ is always larger than nine. To overcome this, we have to extend our construction scheme for $(0,2)$ string models right from the beginning.

\section{Breaking the hidden $E_{8}$}

Geometrically it is clear that one can get much more general gauge groups $G^{\prime}$ by embedding a vector bundle into the so far hidden $E_{8}$ factor, as well. Since this implies that also nonsinglet representations of $G^{\prime}$ are allowed to occur in the massless spectrum, it is possible to reduce the number of vector multiplets considerably by higgsing away parts of the broken gauge group $G^{\prime}$. The question is, whether we can extend the formalism of $(0,2)$ exactly solvable string models proposed in [6] to this general case.

In the spirit of the construction, we describe the embedding of an $S U(2)$ bundle into $E_{8}$ by a spectral flow extension of $S O(12)$ to $E_{7}$. Surely, the spectral flow must have the right 
values $(H, Q)=\left(\frac{1}{4}, 1\right)$ of conformal dimension and $U(1)$ charge. This can be achieved by introducing two $U(1)_{2}$ factors which together with the $S O(12)$ part add up to the correct central charge $c=8$ of the $E_{8}$ current algebra. Thus, we start with the left-right symmetric model shown in Table 4.1.

\begin{tabular}{|l|l|l|}
\hline part & $c$ & $\bar{c}$ \\
\hline $4 D$ space-time, $X^{\mu}$ & 2 & 2 \\
\hline$N=2$ Virasoro & 9 & 9 \\
\hline$U(1)_{2}$ & 1 & 1 \\
\hline gauge group $S O(8)$ & 4 & 4 \\
\hline$U(1)_{2} \times U(1)_{2}$ & 2 & 2 \\
\hline gauge group $S O(12)$ & 6 & 6 \\
\hline
\end{tabular}

Table 4.1 Underlying CFT for $S O(10) \times E_{7}$

The whole partition function is now written in terms of simple current modular invariants in the following way:

$$
\begin{aligned}
& Z \sim \vec{\chi}(\tau) \mathcal{M}\left(\mathcal{J}_{S O(12) \rightarrow E_{7}}\right) M\left(J_{G S O_{L}}\right) \prod_{l} M\left(\Upsilon_{l}\right) M\left(J_{G S O_{R}}\right) \\
& \prod_{i} M\left(J_{i}\right) M\left(J_{S O(8) \rightarrow S O(10)}\right) \prod_{k=1}^{2} \mathcal{M}\left(\mathcal{J}_{S O(12) \rightarrow E_{8}}^{k}\right) \vec{\chi}(\bar{\tau})
\end{aligned}
$$

where the simple currents $\mathcal{J}$ are those new ones which only act on the $\left(U(1)_{2}\right)^{2} \times S O(12)$ piece $^{2}$ :

$$
\begin{aligned}
& \mathcal{J}_{S O(12) \rightarrow E_{7}}=\phi_{1,2}^{U(1)_{2}} \otimes \phi_{1,2}^{U(1)_{2}} \otimes \phi_{s}^{S O(12)} \\
& \mathcal{J}_{S O(12) \rightarrow E_{8}}^{1}=\phi_{2,2}^{U(1)_{2}} \otimes \phi_{2,2}^{U(1)_{2}} \otimes \phi_{0}^{S O(12)} \\
& \mathcal{J}_{S O(12) \rightarrow E_{8}}^{2}=\phi_{1,2}^{U(1)_{2}} \otimes \phi_{1,2}^{U(1)_{2}} \otimes \phi_{s}^{S O(12)} .
\end{aligned}
$$

The last two simple currents guarantee that the right moving side is extended to $E_{8}$ so that the bosonic string map can still be applied to obtain at the end of the day a heterotic string. Unlike those models with a hidden $E_{8}$ gauge symmetry, for these models the leftright symmetry breaking simple currents $\Upsilon_{l}$ are also allowed to contain contributions from the $\left(U(1)_{2}\right)^{2} \times S O(12)$ sector.

${ }^{2}$ For the meaning of our notation and the explicit form of the simple currents $J$ please take a look into [6]. 


\section{Examples}

Now, we are searching for a parent tensor product of $N=2$ minimal models and a set of simple currents $\Upsilon_{l}$ which both yield an $N=2$ space-time supersymmetric spectrum and break the hidden $E_{8}$ down to $E_{7}$. Fortunately, after some failing attempts our computer program provides us with the desired models.

a.) We use the (1) $(4)^{4}$ parent model and choose a certain set of three further $\Upsilon_{l}$ 's yielding the following massless spectrum with gauge group $E_{7} \times E_{7} \times U(1)_{T}^{2} \times S U(3)_{T} \times G(10)$ where only $S U(3)_{T}$ singlets appear in the spectrum: the massless sector is

$$
N_{56,1}=4, \quad N_{1,56}=4, \quad N_{1,1}=72 .
$$

The geometric interpretation is as follows: compactify the ten-dimensional string on a torus down to eight dimensions yielding an enhanced $E_{8} \times E_{8} \times S U(3)_{T} \times U(1)_{T}^{2}$ gauge group. Then embed an $S U(2)$ bundle with $A_{2}^{E_{8}}=12$ into each of the two $E_{8}$ factors and let the toroidal $S U(3)_{T}$ unbroken. The massless spectrum is given by the index theorem (2.1):

$$
N_{56,1}=4, \quad N_{1,56}=4, \quad N_{1,1}=62 .
$$

After higgsing away the $G(10)$ piece we obtain complete agreement of the two spectra.

b.) We find another example of this kind with gauge group $E_{7} \times E_{7} \times U(1)_{T}^{2} \times G(14)$ :

$$
N_{56,1}=2, \quad N_{1,56}=2, \quad N_{1,1}=76 .
$$

Here, we embed an $S U(2)$ bundle with $A_{2}^{E_{8}}=8$ into each of the two $E_{8}$ factors and an $S U(3)$ bundle with $A_{3}^{S U(3)}=8$ into the toroidal $S U(3)_{T}$. The massless spectrum is

$$
N_{56,1}=2, \quad N_{1,56}=2, \quad N_{1,1}=62 .
$$

After higgsing completely the enhanced gauge group $G(14)$ of our model we get exactly this spectrum.

c.) The next example has gauge group $S O(10) \times E_{7} \times U(1)_{T}^{2} \times G(10)$ and

$$
N_{16,1}=8, \quad N_{10,1}=10, \quad N_{1,1}=88 .
$$

After higgsing the $G(10)$ the spectrum agrees with the one obtained by embedding an $S U(4)$ bundle with $A_{4}^{E_{8}}=16$ into the first $E_{8}$, an $S U(2)$ bundle with $A_{2}^{E_{8}}=4$ into the second $E_{8}$ and an $S U(3)$ bundle with $A_{3}^{S U(3)}=4$ into the toroidal $S U(3)_{T}$.

d.) We also obtain a slightly different example with gauge group $S O(10) \times E_{7} \times U(1)_{T}^{2} \times$ $G(8)$ and

$$
N_{16,1}=4, \quad N_{10,1}=6, \quad N_{1,1}=80, \quad N_{1,56}=1 .
$$


After higgsing the $G(8)$ the spectrum agrees with the one obtained by embedding an $S U(4)$ bundle with $A_{4}^{E_{8}}=12$ into the first $E_{8}$, an $S U(2)$ bundle with $A_{2}^{E_{8}}=6$ into the second $E_{8}$ and an $S U(3)$ bundle with $A_{3}^{S U(3)}=6$ into the toroidal $S U(3)_{T}$.

There are many other examples which all have an interpretation in terms of embeddings of suitable bundles into both $E_{8}$ factors. Thus, we have succeeded in formulating the SCFT analogue of choosing arbitrary bundles on $K_{3}$. Clearly, there is no restriction to consider only $N=2$ models so that we claim to have an SCFT analogue of embedding vector bundles on general Calabi-Yau manifolds into both $E_{8}$ factors. This is the general class of $(0,2)$ supersymmetric (non)linear $\sigma$-models studied intensively by J. Distler and S. Kachru in [8]. Furthermore, the generalization to embeddings of higher rank bundles into the second $E_{8}$ is obvious, one starts with $\left(U(1)_{2}\right)^{r} \times S O(16-2 r)$ in the former $E_{8}$ sector and introduces suitable simple currents to extend the sector on the right moving side to $E_{8}$ and on the left moving side to $E_{9-r^{3}}$.

In the following we will investigate example a.) in more detail showing that it gives rise to a lot of possible heterotic/type II dual pairs. By looking at the massless sector we find that actually the fully enhanced gauge group is

$$
G=E_{7} \times E_{7} \times S U(3)_{E} \times U(1)_{E}^{2} \times S U(3)_{T} \times U(1)_{T}^{2}
$$

where the subscript $T$ indicates the gauge factors arising from the torus and the subscript $E$ the special enhanced gauge symmetry. The massless sector fits into the following representations:

$$
4 \times(56,1,1)+4 \times(1,56,1)+18 \times(1,1,3)+18 \times(1,1,1) .
$$

Furthermore, there are singlet fields which carry nonzero charge with respect to the two $U(1)_{E}$ currents. Of course, all states are singlets with respect to the toroidal gauge group. Besides the special enhanced gauge symmetry, this model has already been mentioned in the original paper of S. Kachru and C. Vafa [13] where it was argued that by completely higgsing away the two $E_{7}$ factors and the $S U(3)_{E} \times U(1)_{E}^{2}$ factor one yields a spectrum $\left(n_{H}, n_{V}\right)=(244,4)$. The hypersurface $\mathbb{P}_{1,1,2,8,12}[24]$ has Hodge numbers $\left(b_{21}, b_{11}\right)=$ $(243,3)$ being a good candidate for the type II A dual model. We find further candidates for heterotic/type II A dual pairs in the moduli space of this model.

In Table 5.1 we describe all possible dual pairs which we find in the moduli space of this model by successively breaking parts of the gauge group $G$. The second column shows to which subgroup the gauge group is broken before switching on VEVs for the remaining generators in the $\mathrm{u}$ adjoint representations of the nonabelian sector.

3 We define $E_{5}=S O(10)$ and $E_{4}=S U(5)$. 


\begin{tabular}{|l|l|l|}
\hline$\left(n_{H}, n_{V}\right)$ & $G^{\prime} \subset E_{7} \times E_{7} \times S U(3)_{E} \times U(1)_{E}^{2} \times S U(3)_{T} \times U(1)_{T}^{2}$ & $K_{3}$ fibration \\
\hline \hline$(244,4)$ & $U(1)_{T}^{4}$ & $\mathbb{P}_{1,1,2,8,12}[24]$ \\
$(165,9)$ & $S O(10) \times U(1)_{T}^{4}$ & $\mathbb{P}_{1,1,4,6,12}[24]$ \\
$(128,8)$ & $S O(4) \times S O(4) \times U(1)_{T}^{4}$ & $\mathbb{P}_{2,3,3,16,24}[48]$ \\
$(97,13)$ & $S O(10) \times S O(8) \times U(1)_{T}^{4}$ & $\mathbb{P}_{1,1,6,8,8,10}[1816]$ \\
\hline$(123,11)$ & $S U(4) \times S U(4) \times U(1)_{E} \times U(1)_{T}^{4}$ & $\mathbb{P}_{1,1,4,8,10,12}[2016]$ \\
$(108,12)$ & $S U(5) \times S U(4) \times U(1)_{E} \times U(1)_{T}^{4}$ & $\mathbb{P}_{3,3,4,20,30}[60]$ \\
$(105,13)$ & $S O(10) \times S U(4) \times U(1)_{E} \times U(1)_{T}^{4}$ & $\mathbb{P}_{1,1,6,8,10,10}[2016]$ \\
\hline$(132,12)$ & $E_{6} \times S U(2)_{E} \times U(1)_{E} \times U(1)_{T}^{4}$ & $\mathbb{P}_{1,1,6,8,8}[24]$ \\
$(126,10)$ & $S U(4) \times S U(2) \times S U(2)_{E} \times U(1)_{E} \times U(1)_{T}^{4}$ & $\mathbb{P}_{1,1,4,6,8}[20]$ \\
$(109,13)$ & $S O(10) \times S U(2) \times S U(2)_{E} \times U(1)_{E}^{2} \times U(1)_{T}^{4}$ & $\mathbb{P}_{1,1,6,8,10,12}[2018]$ \\
\hline$(154,10)$ & $S U(3) \times S U(3)_{E} \times U(1)_{E}^{2} \times U(1)_{T}^{4}$ & $\mathbb{P}_{1,1,4,8,10}[24]$ \\
$(112,10)$ & $S O(4) \times S U(2) \times S U(3)_{E} \times U(1)_{E} \times U(1)_{T}^{4}$ & $\mathbb{P}_{1,1,4,6,6}[18]$ \\
$(82,10)$ & $S O(4) \times S O(4) \times S U(3)_{E} \times U(1)_{T}^{4}$ & $\mathbb{P}_{1,1,4,4,6,8}[1212]$ \\
$(63,15)$ & $S U(5) \times S U(4) \times S U(3)_{E} \times U(1)_{E}^{2} \times U(1)_{T}^{4}$ & $\mathbb{P}_{3,3,8,10,24}[48]$ \\
$(40,16)$ & $S O(10) \times S O(10) \times S U(3)_{E} \times U(1)_{T}^{4}$ & $\mathbb{P}_{2,4,4,5,5}^{4}[20]$ \\
\hline
\end{tabular}

Table 5.1 Heterotic/type II A duals

Note that only the first four dual pairs could be found by knowing only the generic gauge group, the entire rest has a nontrivial contribution from the special $S U(3)_{E} \times U(1)_{E}^{2}$ enhanced gauge symmetry. So far we have only dealt with heterotic/type II A duality. However, by looking into the list of [17] we find also some possible heterotic/type II B dual pairs that are presented in Table 5.2. For the type II B string compactified onto a CalabiYau threefold the $N=2$ massless spectrum is $\left(n_{H}, n_{V}\right)=\left(b_{11}+1, b_{21}+1\right)$.

\begin{tabular}{|l|l|l|}
\hline$\left(n_{H}, n_{V}\right)$ & $G^{\prime} \subset E_{7} \times E_{7} \times S U(3)_{E} \times U(1)_{E}^{2} \times S U(3)_{T} \times U(1)_{T}^{2}$ & $K_{3}$ fibration \\
\hline \hline$(42,18)$ & $S O(10) \times S O(10) \times S U(3)_{E} \times U(1)_{E}^{2} \times U(1)_{T}^{4}$ & $\mathbb{P}_{4,9,9,10,22}[54]$ \\
$(35,17)$ & $E_{6} \times S O(10) \times S U(3)_{E} \times U(1)_{T}^{4}$ & $\mathbb{P}_{4,8,10,11,11}[44]$ \\
$(30,18)$ & $E_{6} \times E_{6} \times S U(3)_{E} \times U(1)_{T}^{4}$ & $\mathbb{P}_{8,10,12,15,15}[60]$ \\
\hline
\end{tabular}

Table 5.2 Heterotic/type II B duals

By looking more closely at example b.) we find that the complete gauge group is

$$
G=E_{7} \times E_{7} \times S U(3)_{E} \times S U(2)_{E} \times U(1)_{E}^{3} \times U(1)_{T}^{2} .
$$

The massless spectrum can be reduced out with respect to this gauge group:

$$
\begin{aligned}
& 2 \times(56,1,1,1)+2 \times(1,56,1,1)+4 \times(1,1,3,2)+ \\
& 10 \times(1,1,3,1)+4 \times(1,1,1,2)+14 \times(1,1,1,1) .
\end{aligned}
$$


Despite the fact that the two $E_{7}$ factors minimally can be broken down only to $S O(8)$ we find some further possible heterotic/type II A dual pairs in the different Higgs phases of this model given in Table 5.3.

\begin{tabular}{|l|l|l|}
\hline$\left(n_{H}, n_{V}\right)$ & $G^{\prime} \subset E_{7} \times E_{7} \times S U(3)_{E} \times S U(2)_{E} \times U(1)_{E}^{3} \times U(1)_{T}^{2}$ & $K_{3}$ fibration \\
\hline$(76,10)$ & $S O(8) \times S O(8) \times U(1)_{T}^{2}$ & $\mathbb{P}_{1,1,4,4,6,6}[1210]$ \\
$(77,11)$ & $S O(8) \times S O(8) \times U(1)_{E} \times U(1)_{T}^{2}$ & $\mathbb{P}_{1,1,4,6,6,6}[1212]$ \\
$(47,11)$ & $S O(8) \times S O(8) \times S U(2)_{E} \times U(1)_{T}^{2}$ & $\mathbb{P}_{4,5,5,6,20}[40]$ \\
$(44,12)$ & $S O(10) \times S O(8) \times S U(2)_{E} \times U(1)_{T}^{2}$ & $\mathbb{P}_{6,7,7,8,28}[56]$ \\
$(40,16)$ & $E_{6} \times E_{6} \times S U(2)_{E} \times U(1)_{E} \times U(1)_{T}^{2}$ & $\mathbb{P}_{2,4,4,5,5}[20]$ \\
\hline
\end{tabular}

Table 5.3 Heterotic/type II A duals

Only the last model was already exhibited in Table 5.1. Furthermore, there exist two heterotic/ type II B dual pairs contained in Table 5.4.

\begin{tabular}{|l|l|l|}
\hline$\left(n_{H}, n_{V}\right)$ & $G^{\prime} \subset E_{7} \times E_{7} \times S U(3)_{E} \times S U(2)_{E} \times U(1)_{E}^{3} \times U(1)_{T}^{2}$ & $K_{3}$ fibration \\
\hline$(45,15)$ & $E_{6} \times S O(8) \times S U(2)_{E} \times U(1)_{E}^{2} \times U(1)_{T}^{2}$ & $\mathbb{P}_{5,5,12,16,22}[60]$ \\
$(34,18)$ & $E_{7} \times E_{7} \times S U(2)_{E} \times U(1)_{E} \times U(1)_{T}^{2}$ & $\mathbb{P}_{5,5,6,8,16}[40]$ \\
\hline
\end{tabular}

Table 5.4 Heterotic/type IIB duals

Thus, by studying the enhanced gauge symmetries of only four exactly solvable $N=2$ models we found already 32 different possible heterotic/type II A and 5 heterotic/type II B dual pairs. Since the known lists of $K_{3}$ fibrations are likely far from being complete there might exist even more heterotic strings in the Higgs phases of these models which admit a type II dual description.

\section{Conclusions}

We have studied Gepner-like exactly solvable models with $(0,4)$ world sheet supersymmetry leading to $N=2$ space-time supersymmetric spectra. Unlike the more complicated case with $(0,2)$ world sheet supersymmetry, these models all fit into the scheme of nonlinear $\sigma$-models on $K_{3} \times T_{2}$ with nontrivial embeddings of vector bundles into the gauge degrees of freedom.

In addition, we have extended the class of Gepner-like $(0,2)$ SCFTs to the case where also the hidden $E_{8}$ gauge group is broken down to some subgroup.

Furthermore, by calculating the complete enhanced gauge symmetry of a few examples we have found interesting branches of the heterotic $N=2$ moduli space leading to some candidates of heterotic/type II dual models.

\section{Acknowledgements}

It is a pleasure to thank L. Dolan, S. Kachru and W. Nahm for discussion. This work is supported by U.S. DOE grant No. DE-FG05-85ER-40219. 


\section{References}

[1] G. Aldazabal, A. Font, L. Ibáñez and F. Quevedo, Chains of $N=2, D=4$ heterotic type II duals, hep-th/9510093

[2] I. Antoniadis, E. Gava, K.S. Narain and T.R. Taylor, $N=2$ type II heterotic duality and higher derivative F-terms, Nucl. Phys. B455 (1995) 109, hep-th/9507115

[3] I. Antoniadis and H. Partouche, Exact monodromy group of $N=2$ heterotic superstring, preprint CPTH-RR370.0895, hep-th/9509009

[4] P. Aspinwall and J. Louis, On the ubiquity of $K_{3}$ fibrations in string duality, hepth/9510234

5] P. Aspinwall, Enhanced gauge symmetries and Calabi-Yau threefolds, hep-th/9511171

[6] R. Blumenhagen and A. Wißkirchen, Exactly solvable $(0,2)$ supersymmetric string vacua with GUT gauge groups, Nucl. Phys. B454 (1995) 561, hep-th/9506104

[7] R. Blumenhagen, A. Wißkirchen and R. Schimmrigk, The $(0,2)$ exactly solvable structure of chiral rings, Landau-Ginzburg theories and Calabi-Yau manifolds, preprint IFP-601-UNC, BONN-TH-95-17, NSF-ITP-95-120, hep-th/9510055, to be published in Nucl. Phys. B

[8] J. Distler and S. Kachru, (0,2) Landau-Ginzburg theory, Nucl. Phys. B413 (1994) 213, hep-th/9309110

[9] S. Ferrara, J. Harvey, A. Strominger and C. Vafa, Second quantized mirror symmetry, Phys. Lett. B361 (1995) 59, hep-th/9505162

[10] D. Gepner, Space-time supersymmetry in compactified string theory and superconformal models, Nucl. Phys. B296 (1988) 757

[11] B. Greene, D. Morrison and A. Strominger, Black hole condensation and the unification of string vacua, Nucl. Phys. B451 (1995) 109, hep-th/9504145

[12] K. Intriligator, Bonus symmetry in conformal field theory, Nucl. Phys. B332 (1990) 541

[13] S. Kachru and C. Vafa, Exact results for $N=2$ compactifications of heterotic strings, Nucl. Phys. B450 (1995) 69, hep-th/9505105

[14] S. Kachru, A. Klemm, W. Lerche, P. Mayr and C. Vafa, Nonpertubative results on the point particle limit of $N=2$ heterotic string compactifications, hep-th/9508155

[15] V. Kaplunovsky, J. Louis and S. Theisen, Aspects of duality in $N=2$ string vacua, Phys. Lett. B357 (1995) 71, hep-th/9506110

[16] A. Klemm, W. Lerche and P. Mayr, $K_{3}$ fibrations and heterotic type II string duality, Phys. Lett. B357 (1995) 313, hep-th/9506112

[17] M. Lynker and R. Schimmrigk, Conifold transitions and mirror symmetries, hepth/9511058

[18] A.N. Schellekens and S. Yankielowicz, Extended chiral algebras and modular invariant partition functions, Nucl. Phys. B327 (1989) 673

[19] A.N. Schellekens and S. Yankielowicz, Modular invariants from simple currents. An explicit proof, Phys. Lett. B227 (1989) 387

[20] A.N. Schellekens and S. Yankielowicz, New modular invariants for $N=2$ tensor products and four-dimensional strings, Nucl. Phys. B330 (1990) 103

[21] A.N. Schellekens and S. Yankielowicz, Simple currents, modular invariants and fixed points, Int. J. Mod. Phys. A5 (1990) 2903

[22] N. Seiberg and E. Witten, Electric-magnetic duality, monopole condensation and confinement, Nucl. Phys. B426 (1994) 19, hep-th/9407087; Erratum, Nucl. Phys. B430 (1994) 485

[23] A. Strominger, Massless black holes and conifolds in string theory, Nucl. Phys. B451 (1995) 96, hep-th/9504090

[24] C. Vafa and E. Witten, Dual string pairs with $N=1$ and $N=2$ supersymmetry in four dimensions, preprint HUTP-95/A023, IASSNS-HEP-95-58, hep-th/9507050 